\title{
A case of simultaneous acute generalized exanthematous pustulosis in dizygotic twins
}

\author{
Winston $\mathrm{T}^{\mathbf{1}}$ and Charles $\mathrm{L}^{2}$ \\ ${ }^{1}$ U.S. Military 23rd CBRNE, 2nd Sustainment Brigade, 2nd Infantry Division, Camp Humphreys, Korea \\ ${ }^{2}$ USUHS, Medical Student, 4301 Jones Bridge Rd, Bethesda, MD 20814, Korea
}

\begin{abstract}
Acute Generalized Exanthematous Pustulosis is a rare autoimmune disease that is characterized by sterile pustules amid an erythematous background with accompanied fever and leukocytosis, followed by desquamation of affected skin. AGEP is precipitated by drugs in $90 \%$ of cases, and remaining cases have been reported to be precipitated by infections. To our knowledge, this is the first reported case of dizygotic twins who developed the disease simultaneously. Review of the literature, indicates that genetic tests have identified possible homozygous/heterozygous missense mutations that are responsible for AGEP, however it is unclear whether these are new mutations vs. inherited mutations. Our case suggests that in a portion of the cases, is caused by recessive gene that is inherited.
\end{abstract}

\section{Introduction}

Acute Generalized Exanthematous Pustulosis is a rare autoimmune disease that is characterized by non-follicular sterile pustules on an erythematous background with accompanied fever and leukocytosis followed by desquamation of affected skin [1]. AGEP is precipitated by drug use in $90 \%$ of cases, and the remaining cases have been associated with viral, bacterial and fungal infections (parvovirus B19, cytomegalovirus, coxsackie B4, and Mycoplasma pneumonia), mercury exposure and spider bites [2]. This is the first reported case of dizygotic twins who developed the disease simultaneously. AGEP has an incidence of 1-5 per million [3] and it is extraordinarily rare that two twins would develop it simultaneously. While the pathogenesis and inheritance patterns of this disease are not fully known, genetic tests have identified possible homozygous/heterozygous missense mutations that may be responsible for AGEP [4]. It is unclear whether these mutations are acquired or inherited. Our case contributes to the discussion by suggesting that, in at least a portion of cases, there is a recessively inherited gene.

\section{Case report twin A}

A two-year-old African American male presented to the emergency department with perioral erythema, pustular lesions and scabbing on the back of his neck. He was given steroids and antihistamines with noticeable improvement later in the day. The next day, the erythema spread and he developed pustular lesions on his torso. During the ER clinic follow up, he was given a different antihistamine with a diagnosis of contact dermatitis. One day later he returned to the ER after a dramatic increase in pustular formation periorally and covering and coalescing on his neck, and the flexural portions of his arms and diaper area. He also presented with a fever of $102 \mathrm{~F}$, chills, fussiness, and generalized tenderness to touch. Parents denied recent medication administration, access to medication at home, or any family history of psoriasis (mom however has eczema). Physical exam found full facial edema with erythema, and hundreds of pustules on the face, neck, torso and flexural portions of extremities. No mucosal involvement. Remainder of exam was normal.
Laboratory studies found marked leukocytosis of $19900 / \mu \mathrm{L}$ with neutrophilia $(11270 / \mu \mathrm{L}$; normal $1800-7500)$, monocytosis $(2200 / \mu \mathrm{L}$; normal $<1000)$ and elevated C-reactive protein $(1.8 \mathrm{mg} / \mathrm{L}$; normal $0-.5)$. Liver function test results and urea/creatinine levels were normal. Blood, urine and pustular cultures for bacteria were negative. Serological tests for influenza, respiratory syncytial virus, parainfluenza virus, adenovirus and cytomegalovirus were negative. A skin biopsy found subcorneal spongiform pustules with neutrophilic infiltration. According to the Euroscar study group criteria, our patient had a score of 12, indicating definite AGEP [5]. Our patient was treated with symptomatic care consisting of topical emollients, topical steroids, and pain control. He was also initially treated with IV cefazolin, IV clindamycin and vancomycin for fear of infection until our diagnosis had become finalized. This resulted in rapid resolution of pustules and erythema on day 3 of hospitalization, followed by near full body desquamation (Figure 1).

\section{Case report twin $B$}

A two-year-old boy and fraternal twin of Twin A, two days after his brother's admission, developed perioral edema, mild scabbing, and a few pustules, reminiscent of his brother's early course. He was admitted to the same room as his brother to initiate early treatment, in expectation he would follow a similar course. On admission, physical exam showed a small amount of perioral edema with a few pustules as well as a dozen small pustules on back of neck. He was afebrile and was otherwise had a normal exam. He had an identical HPI as Twin A and followed almost the exact same course as his brother. On day 2.5 of admission, he developed an acute exacerbation of facial swelling, erythema, pustular formation on face, neck, torso and flexural portion

Correspondence to: Winston Turnage, D.O, CPT Winston Turnage, PSC 333 BOX\# 83APO AP 96251-001, Camp Humphreys, Korea, Tel: 010-2844-7216; E-mail: winston.a.turnage.mil@mail.mil

Received: August 28, 2017; Accepted: September 25, 2017; Published: September 28, 2017 


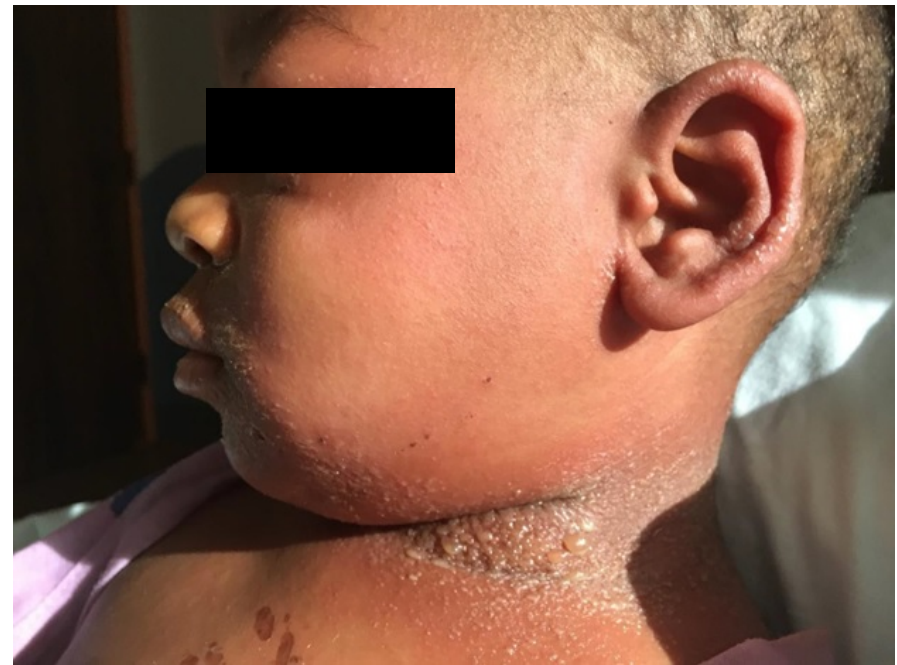

Figure 1. Twin A on day 3 of illness. Full neck, perioral, periocular and tragus pustular formation with facial edema.

of extremities. He remained afebrile throughout his hospital stay. After reviewing the literature, dysfunction of multiple organ systems is rare, however this has been reported 4 and thus we consulted with a pediatric critical care specialist in the event this would occur.

On admission, laboratory studies showed an elevated CRP level (9.9mg/L; normal 0-.5). Liver function studies showed elevated AST (179; normal 5-34) elevated ALT (158; normal 10-53) and urea and creatinine levels were within normal limits. Blood, urine and pustule cultures were negative. Serologic tests for parvovirus B19, Epstein Barr Virus, Influenza A and B, Respiratory Syncytial Virus, parainfluenza virus and adenovirus were negative. The laboratory studies found mild leukocytosis of $10,600 / \mu \mathrm{L}$ with neutrophilia $(8500 / \mu \mathrm{L}$; normal 1800 $7500)$, low eosinophils $(0 / \mu \mathrm{L}$; normal $<800)$, lymphocytopenia $(1600 /$ $\mu \mathrm{L}$; normal 300-9500). These labs were drawn in the resolution phase of his hospitalization. It was our intention to obtain a skin biopsy, however the parents refused due to child distress. According to the EuroScar study group criteria, our patient had a score of [7], indicating probable AGEP. Due to his almost identical clinical course as his twin brother, who had a biopsy confirming AGEP, we feel confident in defining this as AGEP. Our patient was treated symptomatically with pain control, topical emollients, topical corticosteroids, and systemic steroids. Patient had rapid resolution of pustules on day four of admission followed by near full body desquamation (Figures 2, 3 and 4).

\section{Histology}

The histology and immunohistochemistry of biopsy specimens from acute skin lesions of AGEP patients are characterized by a massive cell infiltrate consisting of PMNs in spongiform sub-corneal or intraepidermal pustules, as well as accumulation of CD4 and CD8 T-cells in the subepidermal and perivascular spaces. Often, exocytosis of eosinophils with edema in the papillary dermis is seen and single-cell necrosis of keratinocytes may be present. Expression PMN-attracting chemokine IL- 8 and other factors which activate T-Cell activity, as well as granulocyte and macrophage colony stimulating factors have been found to be elevated in the keratinocytes and infiltrating cells (Figure 5).

\section{Discussion}

Acute Generalized Exanthematous Pustulosis (also known by the names of toxic pustuloderma, pustular drug rash and pustular psoriasiform eruption with leukocytosis) 4 is a rare condition, characterized by the formation of pinpoint, non-follicular, sterile pustules on an erythematous background [6]. As demonstrated with images, our patients developed full body pustules, accentuated in flexural surfaces, and some of which coalesced into bullae.

According to the EuroScar study, antibiotics are the most common cause of AGEP, responsible for approximately 90\% of cases, [7] however non-drug related cases have been reported.1 In children, an acute viral infection is the most common cause of AGEP. The diagnostic criteria set forth by the EuroScar study is a graded criteria consisting of characteristic morphology (pustules, erythema, distribution pattern) followed by post-pustular desquamation with a lack of mucosal involvement. It also takes into consideration acute onset followed by resolution in $<15$ days, fever $>38$ celsius, blood neutrophilia $>7000 /$ ul and spongiform sub-corneal or intradermal blisters (with or without neutrophilic infiltration). 5 Both of our patients simultaneously fulfilled the diagnostic criteria of AGEP.

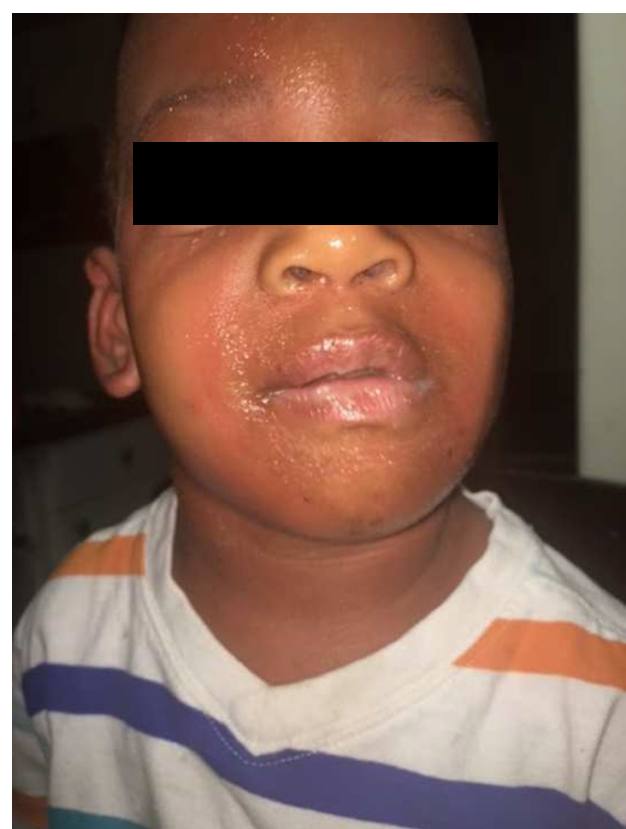

Figure 2. Twin B day one of illness showing periorbital edema and pustules. The patient was admitted at this time to the same room as his brother.

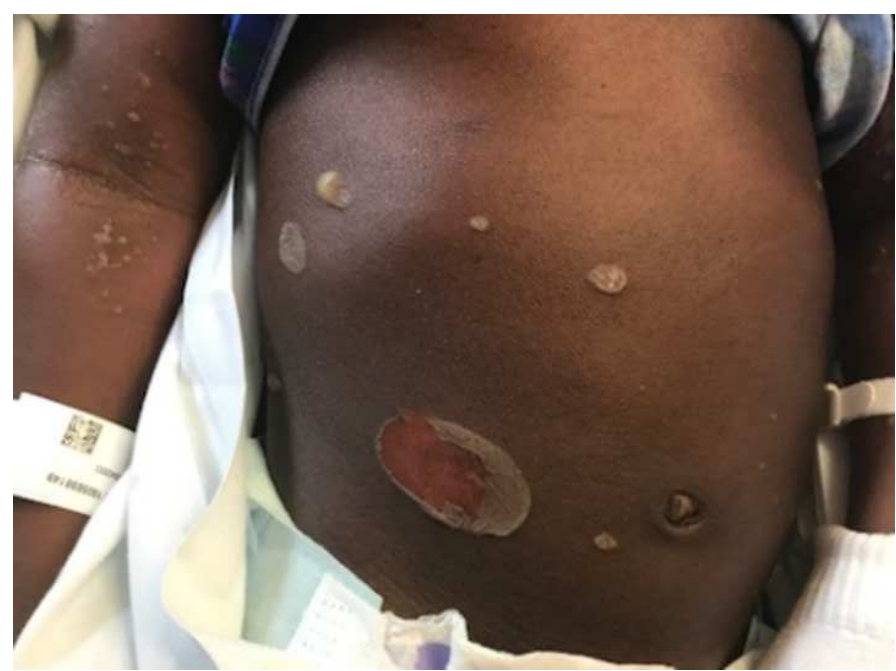

Figure 3. Twin B day 3 acute exacerbation of pustular formation, many coalescing into bullae. 


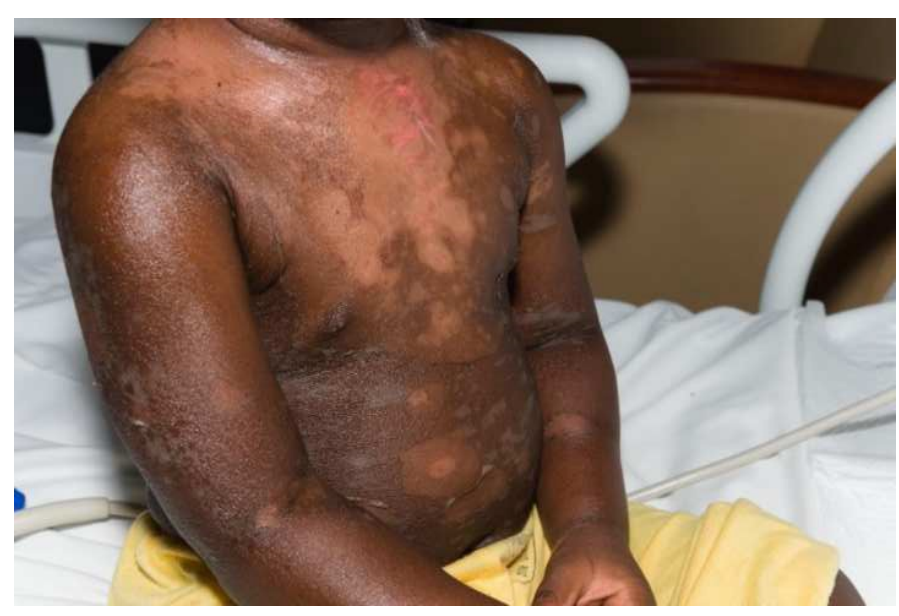

Figure 4. Twin B: Day 6 full body desquamation, however in remarkable good spirits.

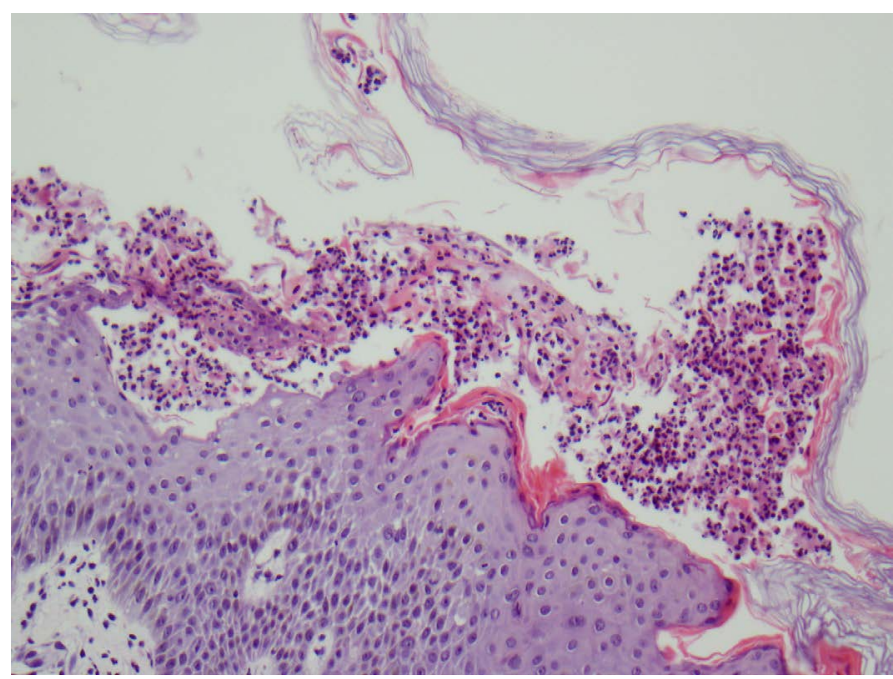

Figure 5. Skin biopsy showing subcorneal pustules with neutrophilic infiltration

The average age of AGEP patients is 56yrs old [4]. It is uncommon in children, and several studies have implicated viruses (EBV, CMV) as a common trigger in children6. The primary differential diagnosis of AGEP is Generalized Pustular Psoriasis (GPP) [8] "GPP shares many of the clinical and immunological findings and the presentation of GPP can appear clinically and histologically indistinguishable from AGEP, and in many cases it is a matter of expert opinion in differentiating the diagnosis. However, AGEP is typically defined by a single episode and self-limiting clinical course, whereas the skin reaction of GPP lasts longer and often recurs" [9]. One must also rule out Stevens Johnson Syndrome and Toxic Epidermal Necrolysis. Stevens Johnson and TEN are characteristically different from AGEP by their slower onset (and longer duration) of 1-3 weeks, blisters instead of pustules, mucosal involvement, and lack of leukocytosis [4].

The pathogenesis of AGEP is not well understood, but the pustules may be a result of T-cell mediated inflammatory response and/or a response to inflammatory cytokines [10]. Current literature to this point in time does not direct a genetic link to this disease however several genetic mutations have been suggested. One study postulates that mutations in the IL36RN gene, which encodes the antiinflammatory IL-36- receptor antagonist, IL-36Ra, is responsible for a sub-segment of AGEP cases. IL-36Ra blocks the pro-inflammatory cytokines IL-36a, IL-36b, and IL-36g. When IL36RN is mutated, IL36 signaling is uncontrolled and enhanced production of IL-6, IL-8, IL-1a, and IL-1b occurs (Onoufriadis et al., 2011), which might give rise to pustular eruptions in AGEP.10 Variations in IL36RN are also common in GPP. IL-8 has further been implicated in AGEP with one study isolating drug specific T-Cells from AGEP patients, with their primary cytokine being IL- 8 . IL- 8 is the cytokine that is responsible for attracting neutrophilic infiltration and resulting in the neutrophilic invasion as seen on histological staining.

In our patients, there is no definitive exposure that we can contribute to their AGEP, however we have three main hypotheses. The first is that these cases of AGEP resulted from a first exposure to tooth cleaning products at a simultaneous first time dentist appointment several days before Twin A developed symptoms. The response could be due to ingredients in the fluoride rinse used by the dentist or due to a transient bacteremia induced by tooth cleaning and micro abrasions in the mouth. Secondly, Twin A had a mild "cough" several days before admission, and both twins could have been exposed to a virus that we did not test for. Finally, the twins recently began using a new bottle of sunscreen. Cases of AGEP have identified the latency period of a reaction to a known source ranging from 1 day due to antibiotics 5 to 44 days for other non-antibiotic sources [11]. Our extensive investigation into the twins' history did not result in a definite culprit, therefore we will define these as cases of idiopathic AGEP.

This unique presentation of twins being affected simultaneously with a very rare disease is an important contribution to the overall knowledge of this disease. The twins are fraternal twins and thus it can be deduced that both parents are carriers of the recessive gene, and that this was not a random mutation.

\section{References}

1. Michael M, Nektaria S, Fani G, Caterina C, Anastasia A, et al. (2009) Acute Generalized Exanthematous Pustulosis (AGEP) Triggered by a Spider Bite. Allergol Int 58: 301303. [Crossref]

2. Birnie AJ, Litlewood SM (2008) Acute generalized exanthematous pustulosis does not always have a drug-related cause. Br J Dermatol 159: 492-493. [Crossref]

3. Sidoroff A, Halevy S, Bavinck JN, Vaillant L, Roujeau JC (2001) Acute generalized exanthematous pustulosis (AGEP)--a clinical reaction pattern. J Cutan Pathol 28: 113119. [Crossref]

4. Leclair MA, Maynard B, Catherine ST (2009) Acute generalized exanthematous pustulosis with severe organ dysfunction CMAJ 181: 393-396. [Crossref]

5. Sidoroff A, Dunant A, Viboud C, Halevy S, Bouwes B, et al. (2007) Risk factors for acute generalized exanthematous pustulosis (AGEP)-results of a multinational casecontrol study (EuroSCAR). British Journal of Dermatology 157: 989-996. [Crossref]

6. Nolwenn R, Laure D (2015) Acute Generalized Exanthematous pustulosis associated with primary Epstein barr virus infection. JAAD Case Reports 1: 9-11. [Crossref]

7. Michael M, Nektaria S, Fani G, Caterina C, Anastasia A et al. (2009) Acute Generalized Exanthematous Pustulosis (AGEP) Triggered by a Spider Bite. Allergology International 58: 301-303. [Crossref]

8. Britschgi M, Pichler WJ (2002) Acute generalized exanthematous pustulosis, a clue to neutrophil-mediated inflammatory processes orchestrated by T cells. Curr Opin Allergy Clin Immunol 2:325. [Crossref]

9. Tyler, Jennifer BS, Stephen S (2012) Two cases of acute generalized exanthematous pustulosis related to oral terbinafine and an analysis of the clinical reaction pattern. Dermatology Online J 18:5. [Crossref]

10. AA Navarini (2013) Rare Variation in IL36RN in Severe Adverse Drug Reaction Manifesting as Acute Generalized Exanthematous Pustulosis. J Invest Dermatol 133: 1904-1907. [Crossref]

11. Beltraminelli HS, Lerch M, Arnold A, Bircher AJ, Haeusermann P (2005) Acute generalized exanthematous pustulosis induced by the antifungal terbinafine: case report and review of the literature. Br J Dermatol 152: 780-783. [Crossref]

Copyright: (C2017 Winston T. This is an open-access article distributed under the terms of the Creative Commons Attribution License, which permits unrestricted use, distribution, and reproduction in any medium, provided the original author and source are credited. 